\title{
Rostros del despojo en Zacatecas
}

\section{Federico Guzmán López}

Durante más de 450 años, el territorio zacatecano ha estado sometido a la explotación y el despojo de su riqueza natural y humana. El origen de la ciudad de Zacatecas, en 1546, se debió al descubrimiento de ricas minas de plata, que permitió el saqueo mineral por parte de los españoles conquistadores. Ahora, el oro, la plata y demás riqueza natural es propiedad de canadiennes, principalmente. Los nuevos rostros del despojo se extienden a la industria manufacturera, los servicios y otros ámbitos de la vida social y cultural.

Primer ARgUMENTO

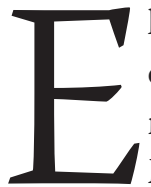
1 extractivismo minero en el estado de Zacatecas tiene gran relevancia económica y territorial. De acuerdo con la Dirección General de Minas, se «tienen un total de 2,537 concesiones mineras, vigentes al 31 de diciembre de 2010, las cuales cubren una superficie de 2'741,067.50 de hectáreas»; ${ }^{1}$ estas cifras equivalen a señalar que hasta 2010 ya se había concesionado $38.05 \%$ del total de la superficie del territorio zacatecano, como se aprecia a continuación en la gráfica 1.
Asimismo, según la Dirección General de Promoción Minera de la Secretaría de Economía, hasta 2010, en México había 732 proyectos con presencia de empresas mineras de capital extranjero, distribuidos en 26 entidades federativas; de los cuales 57 se ubican en territorio zacatecano, cifra que equivale a $7.8 \%$ del total de proyectos a nivel nacional.

De acuerdo a lo anterior, en los próximos años se esperaría un impacto negativo por el despojo a los recursos naturales y al trabajo por las empresas mineras extranjeras instaladas en el estado de Zacatecas. Sin embargo, ya se cuenta con datos reveladores: «las ga- 
GRÁFICA 1

Superficie concesionada a la minería en el Estado de Zacatecas al 2010.

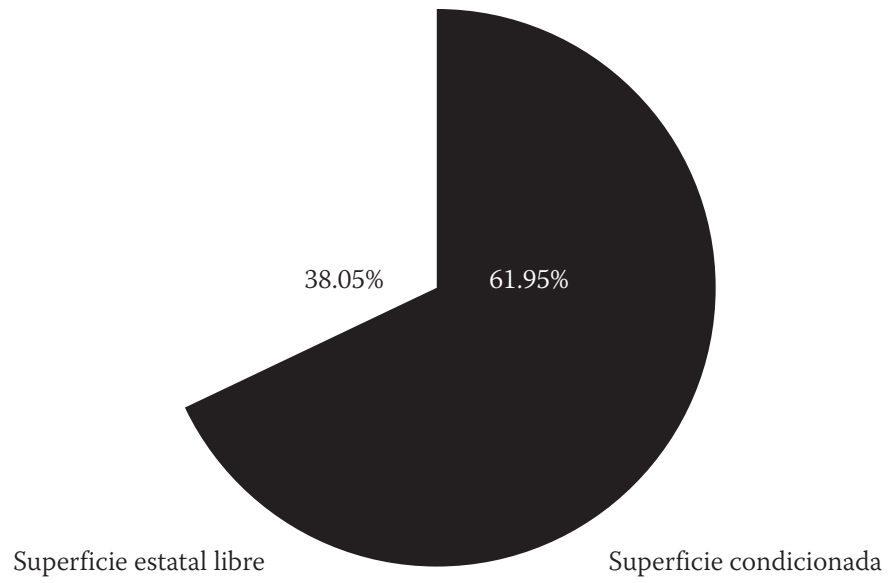

Fuente: Servicio Geológico Mexicano, 2011, p. 11.

nancias de mineras en Zacatecas aumentaron casi 300\% en dos años: sólo en 2009 [...], el valor de la producción minera generada en Zacatecas fue de mil 600 millones de dólares. Para 2011 había aumentado a 4 mil 600 millones de dólares [...] El valor de la producción de 2011 equivale a 2.64 veces al presupuesto de Zacatecas para 2013 (que será de 22 mil 579 millones de pesos, alrededor de mil 736 millones de dólares)». ${ }^{2}$

Cabe preguntarse, ¿por qué el estado de Zacatecas no cuenta con una representación estatal en la Asamblea Nacional de Afectados Ambientales o en la Red Mexicana de Afectados por la Minería? ¿Por qué en Zacatecas no se ha organizado un movimiento de alcance estatal, surgido de la sociedad civil, que genere acciones de resistencia contra la actividad minera? Dicho de una manera más provocadora, quizá habría que preguntarse ¿cuántas toneladas más de oro y plata veremos pasar por nuestras narices, rumbo a Canadá, sin que los pueblos afectados por la minería en Zacatecas, y en general la sociedad civil local, haga algo para detener el saqueo o, por lo menos, para aminorar los efectos económicos, sociales y ambientales del despojo?

De esta manera, se daría un curso distinto a la situación que hace aproximadamente 450 años se heredó desde la época de la colonia, marcada por una relación vertical y de sometimiento que existió entre México y España, situación a la que el estado de Zacate- cas no escapó. Por ello, urge acabar con la somnolencia en la que vive la sociedad civil, misma que, para 2012, se compone de 139 organizaciones, según el Directorio Nacional de Organizaciones de la Sociedad Civil de la Secretaría de Gobernación.

\section{Segundo argumento}

Bajo el amparo de las élites del poder que han controlado el gobierno del estado de Zacatecas en los últimos cuatro sexenios, se ha impulsado como estrategia predominante la promoción de la Inversión Extranjera Directa (IED), en defensa del mito de que «a mayor IED, mayor desarrollo tendrá Zacatecas». En la aplicación práctica de dicho mito se ha dado el mayor y más diversificado despojo en la historia del estado de Zacatecas. En la época colonial, al menos, la minería era principalmente subterránea, menos contaminante que la actual explotación a cielo abierto, y una parte de la riqueza extraída del subsuelo, aunque mínima, servía para edificar las construcciones con valor patrimonial arquitectónico que hoy conforman el Centro Histórico de Zacatecas capital.

Sin embargo, hoy los rostros del despojo están presentes en el extractivismo de recursos mineros principalmente a cielo abierto; en la adicción por el comercio extranjero no sólo 
en bienes de producción, sino también de consumo doméstico, y en la venta de mano de obra barata tanto de las personas que se quedan en Zacatecas a trabajar en las empresas extractivas como de la que emigran a Estados Unidos. Así, Zacatecas vive un momento en el que casi todo se convierte en mercancía, que además se malbarata o se concesiona a muy bajo precio. De esta forma, la fuerza de trabajo, el patrimonio cultural tangible e intangible, el suelo, el agua y la biodiversidad se ponen al servicio del capital transnacional, que le da vida al capitalismo en su modalidad neoliberal en esta entidad.

Hablar de términos como IED, empresas extractivas, cadenas comerciales, capitalismo neoliberal o rostros del despojo puede resultar demasiado abstracto; sin embargo, dichos términos y rostros tienen nombres, por citar algunos: en la minería: Goldcorp y Peñoles; en la industria de bebidas: Grupo Modelo; en
En los hechos, se ha dado el mayor y más diversificado

de Zacatecas. En la época

colonial, al menos, la minería era principalmente subterránea, menos contaminante que la actual explotación a cielo abierto. el comercio y servicios: Soriana, Walt-Mart, McDonald's, Burger King, Samborn's, Oxxo, Extra y la más reciente Plaza Galerías; en las maquiladoras, Delphi Cableados y Yusa; en el sector agrícola, Asgrow, una de las marcas de Monsanto; en el sector del transporte actualmente se debate, en el Congreso local, la concesión de un Metrobús en la zona conurbada Zacatecas-Guadalupe a la inversión privada foránea.

\section{Tercer argumento}

Una mirada al despojo en el contexto nacional lleva a señalar que de acuerdo con datos oficiales, durante el periodo de 1993-2012, en México se otorgaron un total de 44,768 títulos mineros y la superficie concesionada en dicho periodo fue de 97.86 millones de hectáreas, ${ }^{3}$ cifra

GRÁFICA 2

Superficie concesionada a la minería en México en el periodo 1993-2012

(cifras expresadas en millones de hectáreas).

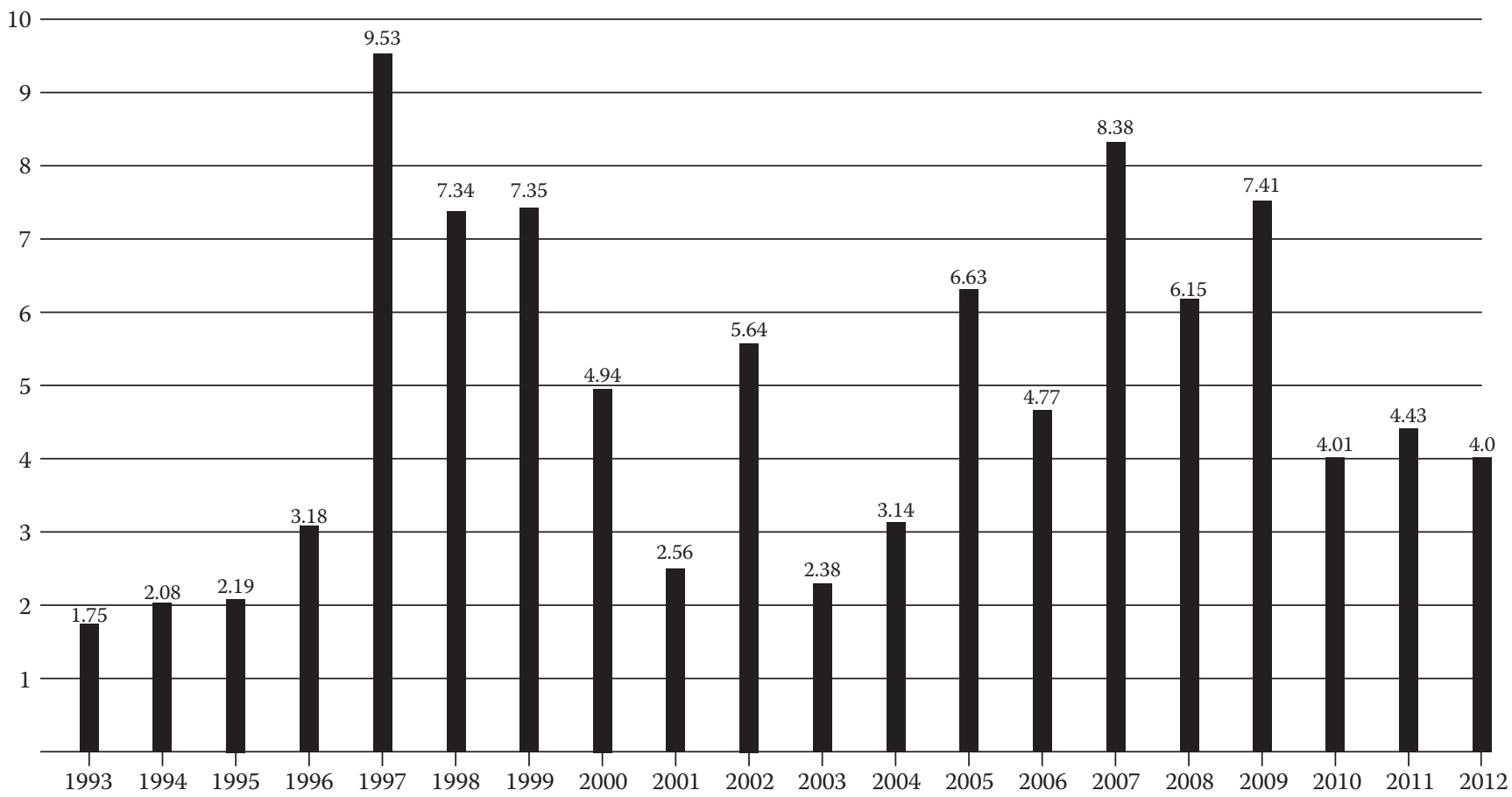

Fuente: basado en SEMARNAT, 2012 
equivalente a $49.8 \%$ del territorio nacional (véase gráfica 2). Asimismo, sólo durante el periodo 2000-2010 la superficie concesionada en México «a las empresas mineras rebasa los 56 millones de hectáreas, espacio que equivale a alrededor del $25 \%$ del territorio nacional». ${ }^{4}$

Ante la dimensión de la problemática antes señalada, la sociedad civil ya se ha manifestado e impulsado acciones de resistencia en contra del despojo y explotación a manos de la «delincuencia de cuello de plata». Ejemplo de ello son la Asamblea Nacional de Afectados Ambientales, organización que tiene presencia en 14 entidades federativas y la Red Mexicana de Afectados por la Minería (REMA), con presencia en más de 24 estados del país.

\section{COMENTARIOS FINALES}

El extractivismo minero se remonta a la época colonial, periodo en que Zacatecas fue despojado de su riqueza minera por España, y ahora resurge con el neoextractivismo de compañías principalmente canadienses. Entre los casos de explotación minera más emblemáticos de la etapa moderna se encuentran la Minera Real de Ángeles, en Noria de Ángeles, Zacatecas, en la década de los ochenta del siglo pasado, y de manera reciente la Minera Peñasquito, en Mazapil, Zacatecas.
Tomando como referentes la manifestación mundial por la paz, celebrada el 15 de febrero de 2003, en la que participaron 15 millones de personas en varios países del mundo, y la más reciente megamarcha organizada por la Unión Nacional de Organizaciones Regionales Campesinas (UNORCA) y Vía Campesina contra Monsanto, un desafío que se lanzaría desde lo local, en el estado de Zacatecas, a la sociedad civil internacional, sería que el 22 de abril de 2013, «Día Mundial de la Tierra», o en otra fecha posterior, la UNORCA y Vía Campesina, en alianza con otras instancias nacionales e internacionales, como la Asamblea Nacional de Afectados Ambientales, la Red Mexicana de Afectados por la Minería, El Barzón, así como las organizaciones participantes del Foro Social Mundial, convocaran a una manifestación mundial en la que participaran todos los pueblos de la tierra afectados por la minería a cielo abierto y por la producción de alimentos transgénicos; como una medida de resistencia en contra de las empresas transnacionales que mediante el despojo controlan la actividad económica en la minería y la agricultura a nivel mundial. Para lo cual se propone que dicha manifestación de escala planetaria, con el lema «Pueblos unidos del sur y norte, todos somos tierra», se realice en defensa de las siguientes demandas: 1) declarar a la tierra como un planeta libre de explotación minera a cielo abierto; 2) revocar concesiones a las transnaciona-

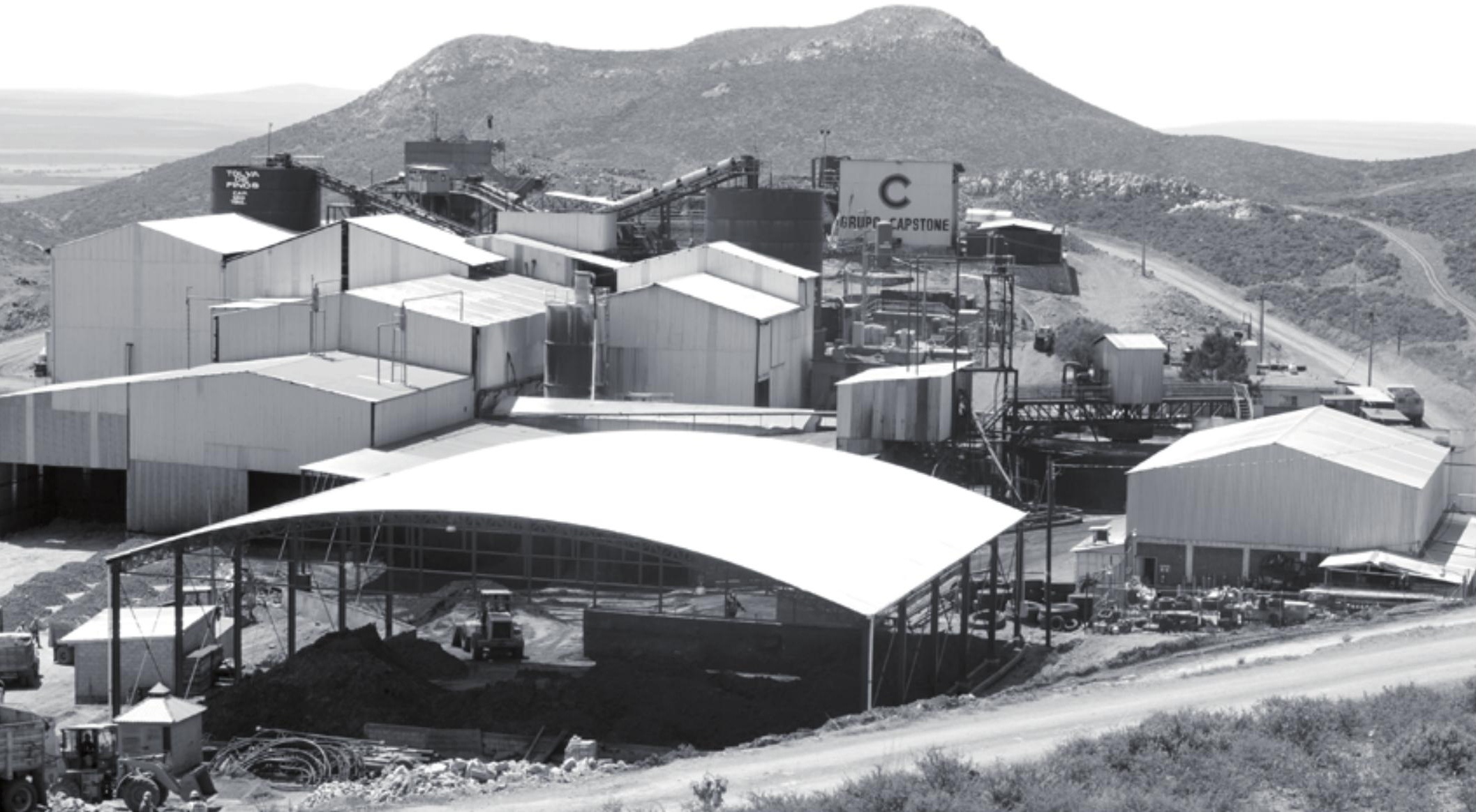


les que realizan su explotación minera a cielo abierto; 3) prohibir la minería a cielo abierto en las Constituciones Políticas de los Estados Nacionales; 4) declarar a la tierra como un planeta libre de producción y consumo de alimentos transgénicos; y revocar licencias de siembra de cultivos transgénicos en todas las naciones del mundo; 5) incorporar la prohibición de cultivos transgénicos en las Constituciones Políticas de los Estados Nacionales, y 6) derogar las leyes nacionales y locales que promueven la explotación minera a cielo abierto y la producción de cultivos transgénicos.

$\mathrm{Al}$ impulsar este tipo de acciones a escala mundial, de las cuales en la Unión Europea ya se tienen avances importantes, también se estaría contribuyendo a proteger el planeta, evitando la contaminación del agua, pérdida de biodiversidad, erosión del suelo y sobre todo evitar pérdidas de vidas humanas en cada país, desde sus grandes ciudades hasta sus pequeñas comunidades.

Independientemente de los posibles alcances que se tuvieran de dicha manifestación, sin duda alguna ello no significaría un impacto inmediato para el desarrollo del estado de Zacatecas. Sin embargo, sería un buen punto de referencia para dar los primeros pasos hacia la posible integración de un actor del desarrollo en Zacatecas (conformado por las organizaciones de la sociedad civil local en alianza con la Universidad Autónoma de Zacatecas), que tuviera como tarea prioritaria la construcción de un proyecto estatal de desarrollo y la responsabilidad colectiva para llevarlo a la acción. Ojalá que durante el posible recorrido de la ruta antes señalada, esta reflexión orientada a la acción también sirviera como primer acercamiento para que el estado de Zacatecas tenga una delegación en la Asamblea Nacional de Afectados Ambientales o en la Red Mexicana de Afectados por la Minería, para coordinar acciones con comunidades de otros estados de la República Mexicana.

Algo debe quedar claro en Zacatecas: ante el extractivismo de la minería y de la industria maquiladora que ya nos llegó, y que en el corto y mediano plazo se avecina a pasos agigantados con mayor intensidad, la sociedad civil local no puede permanecer dormida en el lecho de la indiferencia. No hay que perder la esperanza, pues sí es posible construir un proyecto de desarrollo endógeno que surja desde adentro e impulsado de abajo hacia arriba y de arriba hacia abajo, sin depender de la IED ni de la cooperación internacional.

\section{Referencias}

1 Servicio Geológico Mexicano, Panorama Minero del Estado de Zacatecas, Secretaría de Economía, México, 2011, p. 11.

2 Alfredo Valadez, «Ganancias de mineras en Zacatecas aumentaron casi $300 \%$ en dos años», La Jornada, 4 de febrero de 2013, p. 27.

3 Semarnat, Compendio de Estadísticas Ambientales 2012, México, 2012, disponible en: 〈http://dgeiawf.semarnat.gob.mx:8080/
ibi_apps/WFServlet?IBIF_ex=D2_ENERGIA05_02\&IBIC user=dgeia_mce\&IBIC_pass=dgeia_mce> (consultado el 21 de febrero de 2013).

4 José González, Minería en México. Referencias generales, régimen fiscal, concesiones y propuestas legislativas, Centro de Estudios Sociales y de Opinión Pública, LXI Legislatura, Cámara de Diputados, Documento de Trabajo número 121, México, 2011. 